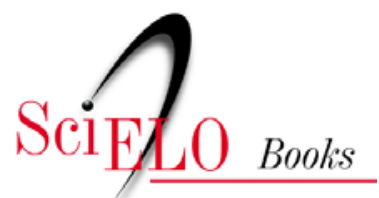

\title{
El trabajo en enfermeira
}

\author{
Maria Consuelo Castrillon Agudelo
}

\section{SciELO Books / SciELO Livros / SciELO Libros}

AGUDELO, M.C.C. El trabajo en enfermeira. In: MACHADO, M.H., org. Profissões de saúde: uma abordagem sociológica [online]. Rio de Janeiro: Editora FIOCRUZ, 1995, pp. 149-162. ISBN: 97885-7541-607-5. Available from: doi: 10.7476/9788575416075.010. Also available in ePUB from: http://books.scielo.org/id/t4ksj/epub/machado-9788575416075.epub.

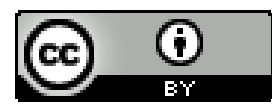

All the contents of this work, except where otherwise noted, is licensed under a Creative Commons Attribution 4.0 International license.

Todo o conteúdo deste trabalho, exceto quando houver ressalva, é publicado sob a licença Creative Commons Atribição 4.0.

Todo el contenido de esta obra, excepto donde se indique lo contrario, está bajo licencia de la licencia Creative Commons Reconocimento 4.0. 


\title{
El trabajo en enfermería
}

\author{
Maria Consuelo Castrillon Agudelo
}

\section{Características generales del trabajo en salud}

El trabajo en salud hace parte del sector terciario de la economía. $\mathrm{Su}$ función social es comparable, en importancia, con el trabajo en el campo de la educación. En el área de la salud se da una fuerte división del trabajo que se ubica en instituciones de diferente naturaleza: sistemas populares, de beneficencia, de seguridad social, atención privada y sistemas oficiales de nivel nacional, departamental o municipal.

El proceso de producir servicios de salud es intensivo en el uso de trabajo humano y de incorporación de tecnología, característica difícil de encontrar en otros servicios. Es trabajo pesado, intenso y extenso en jornada, por eso es de los trabajos más exhaustivos.

La producción y el consumo actúan simultánea y fragmentadamente, es decir, en el mismo momento en que se produce un servicio o un acto en salud, es consumido. Según los economistas, el trabajo es de flujo discontinuo, porque existe un espacio de tiempo entre una actividad y otra. Se producen diversos números de actos o procesos parciales dirigidos a un mismo fin: medios de diagnóstico, actos médicos y de enfermería, trámites administrativos. ${ }^{\prime}$

La división técnica del trabajo en sentido horizontal, se da con mayor intensidad en la profesión médica y da lugar a la especialización que aumenta la complejidad y el costo del acto clínico, por la intervención de un mayor número de profesionales altamente calificados. La especialización conduce a la diferenciación entre productores — en este caso de servicios de salud - que se expresa en tres niveles: a) el de los instrumentos de trabajo: información y equipos: b) el del objeto directo de su trabajo: grupos

1 MEDICI, A. C. Conferencia, Curso Latinoamericano de Recursos Humanos en Salud. Rio de Janeiro, OPS/ENSP-FIOCRUZ, Sep de 1992. 
etáreos, patologías, sistemas, aparatos u órganos: c) el de las acciones técnicas que se desarrollan sobre ese objeto. ${ }^{2}$ Son diferentes los instrumentos de trabajo, el objeto de intervención y las acciones técnicas entre ortopedistas, oftalmólogos y cirujanos generales. Sus actos clínicos generan a la vez intervenciones especializadas por parte del profesional de enfermería. La especialización de los primeros se alcanza por procesos formales producidos por el sector educativo y está regulado por la Ley.

La especialización del profesional de enfermería, se produce regularmente de manera informal, se adquiere durante la experiencia de trabajo en los servicios de salud y opera para efectos legales de responsabilidad profesional y demandas por mala práctica. Esto ocurre por los pocos programas de especialización que se ofrecen, por la ausencia de interés en la dirigencia de las instituciones de salud para especializar este personal, por la falta de proyectos de desarrollo y cualificación para el personal vinculado a los servicios de salud y, en especial, porque la mayor calificación significa contar con mayor presupuesto para crear cargos y ajustar salarios. Las mismas razones explican los motivos por los cuales se han dificultado los proyectos para profesionalizar auxiliares de enfermería.

La especialización adquiere legitimidad en el nivel terciario - de alta tecnología y mayor complejidad hospitalaria- según la división de la atención en salud y en grandes concentraciones urbanas, donde se ubica la mayor parte de los profesionales del área. Otra situación ocurre en el primer nivel de atención y en los programas de atención primaria dirigidos a poblaciones rurales y marginadas urbanas. Los informes presentados al XVI Congreso Cuadrienal del Consejo Internacional de Enfermeras, realizado en Japón en 1976, son elocuentes y dan cuenta de una realidad que veinte años después permanece sin modificaciones:

Es sin duda la insuficiencia de médicos en zonas rurales alejadas $y$ en zonas urbanas donde se vive en condiciones de indigencia o de pobreza, la causa mayor que lleva a considerar a la enfermera como asistente sanitario primario ${ }^{3}$

En Tailandia, las enfermeras pueden legalmente desempeñar funciones curativas en clínicas rurales donde no hay médicos ${ }^{+}$

2 DONNANGEI.O, M. C. Medicina e Sociedade. São Paulo, Pioneira, 1979.

3 SANDOVAI, I. Enfermería en América del Sur y Central. EN: Anec. Año VII (21-22): 32-41, May-Dic 1977.

4 DE SILVA, H. Enfermería en el Sudeste Asiático. EN: Anec, Año VII (21-22): 25-31, May-Dic 1977. 
Bajo esta racionalidad de servicios insuficientes para poblaciones pobres, se desarrolla la política de ampliación de coberturas, apoyada en personal de enfermería de diferentes niveles y en promotores de salud. En muchos lugares, la atención del parto está relacionada con la capacidad de pago de la usuaria del servicio, determinando la atención por un obstetra, una enfermera o una partera empírica.

Por otra parte, existe una división técnica del trabajo, que opera verticalmente, a partir de la redistribución de tareas entre personas de diferentes niveles de calificación, que genera una importante demanda de personal auxiliar ${ }^{5}$ por ser menos costoso para un sector de carencias seculares. Este fenómeno tiene mayor presencia en enfermería, donde existen al menos tres niveles jerárquicos entre: profesionales, auxiliares y ayudantes de enfermería -o atendentes como se les llama en algunos países. En todo trabajo institucional se debe atender a la normatización de las actividades, la racionalización del uso de instrumentos de trabajo, el establecimiento de vías institucionales de cooperación entre el grupo o equipo de trabajo. Allí se ubican resistencias de los profesionales y técnicos para someterse a normas capaces de interferir con su autonomía. ${ }^{6}$

En relación a la labor ejercida por los trabajadores de la salud, el trabajo médico posee la máxima autonomía y ejerce gran poder institucional. Los profesionales de enfermería realizan las coordinaciones con las áreas de apoyo y viabilizan el cumplimiento de normas y rutinas en los servicios, situación que puede generar conflictos intergrupales. Estos cruces de poderes dificultan la mayoría de las veces la convivencia, la tolerancia, el ejercicio de la democracia y el respeto de los derechos de los enfermos y demás usuarios de estos servicios.

\section{El trabajo en enfermería}

El trabajo en enfermería es acentuadamente estratificado por niveles de formación, títulos variables por países y escasa delimitación de las acciones desarrolladas por cada categoría ocupacional. El predominio de mujeres en su composición, en un mundo donde el poder es masculino, ha construido una cultura de trabajo remedo de la familia, donde la au-

5 BELMARTINO, S. et al. El mercado de trabajo médico y la producción de servicios de salud en Argentina. EN: Cuadernos Médico Sociales. (20): 10, Jun. 1986.

6 BELMARTINO, S. et al. Ibid. p. 11. 
toridad paterna se atribuye al médico y el rol de madre sumisa y obediente a la enfermera.

En el campo hospitalario, la enfermera tiene escasa autonomía técnica y administrativa, en el comunitario requiere de iniciativa y creatividad para acompañar proyectos sanitarios y tomar decisiones de todo tipo.

La historicidad del proceso de división del trabajo en enfermería, muestra una realidad diferente a las aspiraciones que la idealizan, tanto en su función social de cuidar, como en la de administrar el cuidado, o gerenciarlo -como se denomina actualmente -. Por historicidad del proceso de trabajo se entienden las transformaciones que ocurren tanto en el objeto y finalidad del proceso, como en la formación, denominación y actividades desempeñadas por los agentes -en este caso las enfermerasy sobre todo en el cuerpo de conocimientos que preside el proceso.'

La enfermera, en los actuales servicios de salud, se ha distanciado de las actividades correspondientes a su preparación técnica y ha asumido actividades dispersas en: administración de personal, administración de recursos materiales, alimentación de sistemas de información, tareas que no realiza otro personal. Esta situación ha sido constatada por diferentes estudios, uno realizado por antropólogos, en Buenos Aires - Argentina -, refiere:

La amplia gama de funciones que debe cumplir: distribución de trabajo al personal: disposición del personal en los distintos dias, turnos y servicios: consecución de ropas, materiales, medicamentos y aparatos: puesta al día de información y ficheros: atención a las demandas de los familiares de los pacientes: coordinación de los servicios de diagnóstico y tratamiento: atención de los pacientes, etc., diluye el perfil de su rol y la dispersa en un sinnúmero de tareas que se apartan del objetivo mismo de la enfermería ${ }^{8}$

Otro estudio, concluye que los profesionales de enfermería, cuya formación tiene un alto costo social, así como un alto nivel de conocimientos, actitudes y aptitudes, están subutilizados por las instituciones de salud y marginados totalmente en su ejercicio profesional con graves consecuencias tanto en la calidad de la atención en salud, como en satis-

7 ROCHA, S. M. M. : ALMEIDA, M. C. P. Origem da enfermagem pediatra moderna. EN: Revista Escola de Enfermagem da USP. Vol. 27 (1): 26, abr. 1993.

8 PIÑA, Nelly: DAINO, Leonardo y MORENO, Inés. La inserción de enfermería en el contexto hospitalario. EN: Anec, Vol. VII (20): 17-33. Ene-Abr. 1977. 
facción profesional. ${ }^{9}$ Razones para esta situación se ubican en la insuficiencia de este personal, demostrada por estudios en diversas instituciones y países. Influyen también, la flexibilidad institucional para asignar e inventar funciones de acuerdo con las necesidades de cada momento y el contraste que existe entre la amplia gama de especialidades médicas y las restringidas oportunidades de especialización que tienen las enfermeras vinculadas a los servicios de salud.

Sin un referente conceptual de enfermería, que fundamente su ejercicio profesional, es imposible determinar su área de responsabilidad. Cada enfermera la identificará de diferente forma y, en consecuencia, desarrollará las actividades que considere necesarias. De ahí la importancia de recuperar la historicidad del cuidado, analizar críticamente su pertinencia y resituarlo como objeto del saber y la acción del profesional de enfermería.

La falta de una identificación y apropiación de su objeto de trabajo y de su objetivo social, así como la división del trabajo entre diferentes niveles de enfermería, genera contradicciones entre rescatar el cuidado directo o fomentar las acciones gerenciales. Esto desemboca en conflictos en relación con la formación profesional y en la definición de su papel, tanto dentro del equipo de enfermería como en el multiprofesional del campo de la salud, "' limita el desarrollo del conocimiento, frena las posibilidades de una configuración profesional de mayor solidez y priva a la población de recibir atención en salud de bajo riesgo.

La ausencia de consenso profesional acerca de sus funciones y actividades, dificulta el establecimiento de una política asistencial de enfermería en cualquier institución de salud. Además, el extenso campo de acción en que debe desenvolverse estos profesionales, afecta no sólo la coherencia interna de sus conductas, como también divide al grupo humano constituido por sus pares, creando fracturas artificiales que les impide actuar organizadamente para reconstruir y reorientar sus perfiles profesionales.

En la práctica hospitalaria, no se ha logrado la conquista idealizada de la autonomía profesional, porque sus actuaciones y decisiones quedan entre la autoridad de una administración superior y la autoridad técnica

9 CASTRILION, M. C.; ESCOBAR, I. y PUI IIDO, S. Calidad de Atención de enfermería en salas de trabajo de parto. Informe de Investigación, Universidad de Antioquia, Facultad de Enfermería, 1990.

10 KURGANT et al. Percepção dos enfermeiros sobre as atividades que desenvolvem em suas unidades de trabalho. EN: Revista da Escola de Enfermagem da USP. Vol. 27 (2): 229-245, Ago. 1993. 
del médico que, como ya se mencionó, posee la máxima autonomía en relación a los demás profesionales del campo de la salud.

En enfermería comunitaria, existen condiciones para desarrollar un modelo profesional autónomo y de mayor impacto en la gestión y acompañamiento de proyectos de salud participativos, en los campos de la promoción de la salud y la prevención de enfermedades. Pero es este espacio el que concentra menor presencia de profesionales y mayor dispersión, donde se requiere una mayor fundamentación en el conocimiento de las relaciones salud-sociedad, que no proporciona la formación de pregrado y, donde las presiones políticas se imponen sobre la racionalidad técnica a la hora de tomar decisiones.

\section{Condiciones de trabajo}

Por condiciones de trabajo se entiende el conjunto de factores que actúan sobre el individuo en el medio laboral. En otras palabras, son las características o modalidades bajo las cuales se consume la fuerza de trabajo - energía humana, física y mental empleada en el proceso -.

En la producción social de bienes y servicios necesarios para el desarrollo de la sociedad en su conjunto, el trabajo constituye la actividad básica mediante la cual los individuos se incorporan a la producción y a los servicios y conforman grupos con características socio-económicas específicas, que guardan relación con las condiciones bajo las cuales trabajan y con el modo de vida o condiciones bajo las cuales recuperan la fuerza física y mental consumida para mantener su capacidad de trabajo.

Así se han diferenciado en lo teórico metodológico, para fines de investigación en el terreno de las relaciones salud-trabajo, el momento de la producción - durante el trabajo - y el de reproducción - durante el consumo de bienes."

Estudiosos del tema diferencian condiciones de trabajo intrínsecas y extrínsecas. Las condiciones intrínsecas, se refieren al trabajo propiamente dicho: al interés intelectual que representa la tarea: al tipo de trabajo: a las posibilidades de desarrollo personal, contenido, integralidad y cualificación del trabajo. Las condiciones extrínsecas pueden ser de orden material, estar ligadas a la organización o pertenecer al nivel

11 Ver la producción en medicina social, de las Escuelas de Quito y Xochimilco, en el campo de condiciones de vida y salud y de las relaciones salud y trabajo, en particular las propuestas teórico-metodológicas de Jaime Breilh y Cristina Laurell. 
psicosocial. Entre las de orden material se cuentan los aspectos de higiene y seguridad, comodidad y espacio físico. Se relacionan con la organización: las políticas institucionales, horarios de trabajo, salarios y estabilidad del empleo. Las psicosociales corresponden a las características del trabajo, relaciones horizontales y verticales: características del mando: información y canales de comunicación. ${ }^{12}$

El conjunto de acciones de salud que conforman la respuesta social frente a los problemas sanitarios, constituye un aspecto de la producción de servicios, donde el proceso de trabajo, particularmente el de enfermería, se caracteriza por una serie de actividades y tareas de procesos discontinuos, con grados de responsabilidad y complejidad diferentes según su relación con el tipo de función dentro del complejo engranaje que comprende a la producción de servicios de salud.

Las condiciones de trabajo de las enfermeras pueden caracterizarse de altamente desgastantes por: sobrecarga de tareas, jornadas prolongadas - a veces de doce horas - turnos rotatorios, trabajo nocturno ${ }^{13}$ : frecuentes cambios entre áreas de diferente especialidad, escasas horas de descanso y carencia de lugares apropiados para tal fin dentro de los servicios; ${ }^{1+}$ pocas posibilidades de ascenso y promoción ${ }^{15}$. Además, porque es un personal expuesto permanentemente a riesgos biológicos - virus, bacterias, hongos - químicos en todos los estados, que representan riesgos dermatológicos, oncológicos y para la salud reproductiva: físicos, que se constituyen en riesgos para accidentes, trastornos osteoarticulares y oncológicos. Más recientemente, se suma el riesgo de perder la vida, si se trabaja en servicios de urgencias o de cirugía, o en trabajo de campo en regiones convulsionadas por la violencia de diferente orden.

En este ámbito de trabajo - el de los servicios de salud - la percepción individual de la muerte, el dolor y el sufrimiento, ya no se manifiesta a distancia como puede ocurrir en el transcurso de la vida cotidiana. Adquiere una presencia que marca diversas formas de asumir la propia vida.

12 CORREA, A. E. et al. Congelación de la Planta de Cargos y su relación con las condiciones de trabajo. Clínica León XIII, ISS, Antioquia. Informe de Investigación. Universidad de Antioquia-Anec-Iss, Medellín, 1990.

13 ARANGO, E. et al. Características socio-económicas y condiciones de trabajo de enfermeras egresadas de la Universidad de Antioquia. EN: Investigación y Educación en Enfermería. Vol. III (1): 23. Mar. 1985.

14 CASTRILLON, ESCOBAR y PULIDO. Opus. Cit. p. 25.

15 CORREA, A. E. et al. Opus Cit. p. 3. 
Finalmente, las carencias institucionales, en recursos materiales y humanos básicos, en suministro y mantenimiento oportuno de equipos y en la aplicación de normas científico-técnicas actualizadas, contribuyen al desgaste laboral de todo el personal de salud, deterioran progresivamente los servicios de salud, en especial los estatales, impactan la calidad de la atención y someten a riesgos permanentes a los usuarios de los servicios. ${ }^{16}$

\section{El proceso de trabajo en enfermería}

El proceso de trabajo, entendido como la forma histórica y social de organizar la actividad laboral, conjuga recursos de diverso orden -financiero, material, tecnológico, humano-. El proceso de ofrecer servicios de salud en general y de enfermería en particular, se inscribe en un medio institucional que lo condiciona mediante múltiples determinaciones, entre otras, la cantidad, calidad, y modalidades de distribución de los recursos: grado de complejidad de los problemas de salud que se deben atender y relaciones científico-técnicas, políticas y afectivas, derivadas de la distribución social de saberes, poderes y formas de comunicación interpersonal.

Según algunos autores, ${ }^{17}$ el proceso de trabajo en salud se reorganizó alrededor de dos ejes básicos: 1) el control de la enfermedad en escala social: 2) la recuperación de los enfermos a nivel individual.

Sobre el primero se generaron modelos de organización de acuerdo con el énfasis conceptual y político hacia la enfermedad o hacia la salud. Así, se han desarrollado variados instrumentos de trabajo para diagnosticar situaciones de salud en poblaciones, levantar sus perfiles epidemiológicos o identificar necesidades de salud -según el enfoque-, así como para planificar o intervenir los ambientes de vida y de trabajo, educar para la salud y desarrollar acciones tendientes a la prevención de enfermedades y promocionar la salud y las condiciones de vida saludables.

El segundo proceso, está determinado por el paradigma clínico, centrado en la enfermedad, con sede en el cuerpo humano. El sujeto de intervención puede ser el humano enfermo o su patología - según la concepción que se tenga. El objetivo final, restaurar la salud o hacer

16 CASTRILLON, M. C. Deterioro de los servicios de salud en un contexto de crisis. Trabajo Libre presentado durante el V Congreso Latinoamericano de Medicina Social. Caracas, Marzo de 1991. Resumen EN: Memorias del Congreso, p. 329.

17 BRITO, P.; NOVICK, M. y MERCER, H. Personal de salud y trabajo: Una mirada desde las Instituciones. Rio de Janeiro, Documento Clarhus, Ops/Ensp-Fiocruz. Ago. 1992. 
menos traumática la muerte. Se trabaja con instrumentos científico-técnicos y administrativos, para el diagnóstico, tratamiento y cuidados que demande la situación de cada sujeto de atención, en una organización mixta que conjuga procesos de alta tecnología en medios diagnósticos y terapéuticos, con procesos manuales de tipo artesanal.

Los dos procesos son altamente conflictivos, por el uso intensivo de trabajo humano, donde es necesaria la interrelación de actores diversos cargados de valores, símbolos, representaciones y poderes frente a la salud, la enfermedad, la vida, la muerte y las identidades profesionales, factores que se han acumulado a lo largo del tiempo hasta conformar una compleja cultura organizacional.

El trabajo en equipo multiprofesional es fundamental en la atención en salud, debido a que en el individuo o en el colectivo se concentran numerosas acciones sanitarias que requieren interpretación, coordinación e integralidad para que las intervenciones sean efectivas.

El profesional de enfermería es un componente básico del equipo sanitario, sus funciones y actividades están íntimamente ligadas con las desempeñadas por otro personal. En su constitución histórico-conceptual, el cuidado ha sido objeto de su saber y de su práctica, pero tal realidad no está en la conciencia de todos los profesionales. La división social y técnica del trabajo atomiza y dificulta en la práctica una visión global de la especificidad de sus intervenciones y la construcción de un proyecto de trabajo que permita pensar y actuar organizadamente en la búsqueda de fines específicos en salud.

Por eso, es necesario reflexionar, discutir y clarificar acerca de las relaciones y diferencias entre funciones asistenciales y gerenciales del trabajo profesional en enfermería. ${ }^{18}$ Hoy se observa que la asistencia directa es ejercida por todos los miembros del grupo de enfermería, en mayor proporción por personal auxiliar. La planeación, dirección y control de tales actividades se supone a cargo de un profesional que dada la insuficiencia cuantitativa, no puede hacer la cobertura espacio-temporal que tales responsabilidades demandan.

Gerenciar el cuidado y los servicios de enfermería, no constituye un desvío de su función esencial: lo es cuando se distorciona la administración adoptando tareas dispersas que nadie realiza, por temor a enfrentar

18 SILVA, GOMEZ y ANSELMI. Enfermagem: Realidade e perspectiva na asistência e no gerenciamiento. EN: Revista Latinoamericana de Enfermería. Año 1, Vol. 1: 59-63. Janeiro, 1993. 
con seriedad administraciones erráticas y el desorden institucional: o cuando la profesión se asume como servicio de la profesión médica.

Las enfermeras desarrollan sus actividades profesionales en forma rutinaria, fundamentadas en la tradición y en la autoridad y no en la reflexión sobre su práctica. ${ }^{19}$

A partir del momento en que el profesional de enfermería centre su atención en su objeto de trabajo - el cuidado de la salud individual o en colectivos - y cree un proceso de trabajo integrado con el equipo de enfermería, habrá ganado un importante terreno en el proceso de recobrar su identidad profesional, de cualificar la prestación de servicios de salud y podrá asumir desde una ética civil la responsabilidad de sus decisiones y de sus actos profesionales. Para que ésto ocurra, será necesario que el profesional de enfermería deje de llenar papeles que no son de su responsabilidad, de atender el teléfono, de buscar que los servicios de apoyo respondan eficientemente a las demandas de los servicios y abandone la preocupación por los inventarios y el mantenimiento de equipos -asuntos que competen a la administración general.

Algunas autoras, dicen que la especificidad del trabajo de la enfermera, está dirigida por dos ejes centrales: la asistencia, que constituye el trabajo directo de cuidado, en una relación interpersonal: y la organización de esta asistencia, que se realiza mediante el proceso administrativo. ${ }^{20}$ Otros planteamientos postulan que esas dos orientaciones no constituyen polos opuestos del trabajo de la enfermera, por el contrario hacen parte de un mismo proceso de trabajo. Quienes así piensan, señalan que la dicotomía entre administrar y asistir no deriva de la práctica profesional sino del discurso que se construye en el ámbito de lo académico, que no se apoya ni se legitima en la realidad del trabajo de la enfermera y de la salud como un todo. ${ }^{21}$

19 KURGANT, M. C. et al. Percepção dos enfermeiros sobre as atividades que desenvolven en suas unidades de trabalho. EN: Revista da Escola de Enfermagem da USP. Vol. 27 (21): 229, Ago. 1993.

20 CARRASCO, M. A. P. Influência das escolas de administração científica e clássica na produção científica da enfermagem brasileira. EN: Rev. da Escola de Enfermagem da USP. Vol. 27 (1): 43-65, Abr. 1993.

21 CARRASCO, M. A. P. Influência das escolas de administração científica e clássica na produção científica da enfermagem brasileira. EN: Rev. da Escola de Enfermagem da USP. Vol. 27 (1): 43-65, Abr. 1993. 
Se necesita abandonar la rutina que se sigue en la actualidad, retomar herramientas disponibles y, en lo posible, crear nuevas maneras de ser, hacer y pensar enfermería. Entre las herramientas disponibles están: el proceso de enfermería y el administrativo, además de la orientación al trabajo interdisciplinario y la mayor conciencia de los nefastos efectos que puede dejar la atención de salud deficiente, asunto que hace referencia a la calidad de la atención.

\section{El trabajo interdisciplinario}

El trabajo interdisciplinario no es un formalismo, constituye una práctica de integración social participativa y democrática, un trabajo colectivo donde se comparten objetivos, conocimientos y experiencias para ofrecer una solución integral a problemas de salud de individuos y de colectivos. Para lograrlo se necesita que cada actor tenga claridad sobre su campo de intervención, sus posibilidades y limitaciones, y sobre el campo de los demás. De igual manera, es necesario reconocer y respetar la diferencias de saberes, género y profesiones, potenciar la capacidad de cada actor, reconocer su importancia dentro del grupo de trabajo en el sentido de que su conocimiento y participación aporta a la solución de problemas determinados.

En la estructura interna de un equipo de salud en general y de enfermería en particular se deben conocer los perfiles profesionales, las funciones y responsabilidades de cada uno: compartir información y resultados: resituar los problemas en el conjunto de la estructura y la organización del trabajo, en una permanente comunicación.

Que un equipo técnico se denomine interdisciplinario, no garantiza que funcione como tal. Tampoco los equipos trabajan interdisciplinariamente como consecuencia de alguna norma. Sólo la toma de conciencia de la necesidad de trabajar en forma armónica, más horizontal y recíproca, puede conducir a tal meta.

\section{Calidad de atención de enfermería}

En la medida en que los servicios se amplían y el derecho a la salud se hace vigente, se vuelve indispensable garantizar la calidad de la atención, porque el compromiso por elevar las condiciones de vida y de salud 
de la población es inseparable del compromiso ético de asegurar la calidad de los servicios.

Muchos aspectos se han sugerido, como componentes de la calidad de la atención en salud, entre otros: accesibilidad física, económica y cultural: idoneidad en el sentido de actualidad científico-técnica: coordinación entre profesionales y técnicos dentro de la institución: efectividad en tanto condiciones del servicio para satisfacer las necesidades requeridas: eficacia para producir el efecto deseado con el mínimo de esfuerzos y gastos: participación del enfermo y su familia en la toma de decisiones y respeto por tales decisiones: seguridad en el medio asistencial y oportunidad en la asistencia.

La utilización adecuada de recursos, el porcentaje de infecciones, las tasas de reingresos, las complicaciones, las muertes evitables y muchos otros son parámetros que se deben monitorizar para corregir y mejorar la asistencia. ${ }^{22}$

También se han tenido en cuenta: la suficiencia y calidad de los recursos físicos y materiales: la disponibilidad y capacidades profesionales y técnicas del recurso humano según el nivel de atención: el suministro de material de consumo, el mantenimiento preventivo de equipos: la organización de los servicios: la realización de los procedimientos y actividades de atención conforme a las normas científico-técnicas vigentes y el trato social y humanitario.

La calidad de la atención puede enfocarse desde tres ángulos diferentes: en términos de satisfacción de las expectativas del usuario, de la institución y del trabajador de la salud. ${ }^{23}$ El paciente generalmente enfatiza su relación con el personal de salud, la oportunidad y claridad de las informaciones que recibe, la oportunidad en la atención, la alimentación y comodidad. Los trabajadores de la salud manifiestan insatisfacción por el salario que perciben, la inadecuación de las áreas de trabajo, el insuficiente apoyo institucional, la ausencia de planes de desarrollo que les permita capacitarse para un mejor desempeño. La institución evalúa la consecución de objetivos mediante indicadores de resultados que pueden ser la disminución en las tasas de mórbilidad y de mortalidad o el número de acciones realizadas por período de tiempo.

22 ESTEBAN, A. Perspectiva de los programas de calidad de asistencia. EN: Todo Hospital. (80): 13-16, Oct. 1991.

23 AGUIRRE-GAS, H. Evaluación de la calidad de la atención médica. Expectativas de los pacientes y de los trabajadores de las unidades médicas. EN: Salud Pública de México. 32 (2): $170-180$. 
Para medir la calidad de atención de enfermería, William Holzemer (enfermero norteamericano), ha sistematizado una propuesta para estudiar procesos particulares en el área clínica. Adaptando la propuesta de este autor y la de otros, en especial la del doctor Avedis Donabedian, se podría proponer una serie de indicadores referentes a los tres objetos de observación: el enfermo, las acciones científico-técnicas del profesional de enfermería y el contexto institucional.

En cada uno se estudiarían: a) condiciones de entrada, por ejemplo, las condiciones bio-psico-sociales del enfermo, estructura del servicio y condiciones de trabajo del profesional de enfermería: b) desarrollo del proceso: evolución del enfermo, aplicación del proceso de enfermería y operatividad técnico-administrativa de la institución: c) resultados en: condiciones de egreso del paciente, evaluación del plan de atención de enfermería y objetivos institucionales. El análisis cualitativo de esta red de procesos permitiría aproximar un juicio sobre la calidad de la atención. Algunas experiencias se han realizado con resultados preocupantes, que deben llamar la atención del personal de enfermería y de las instituciones de salud por la tendencia creciente al deterioro de los servicios.

En el terreno de la salud pública, se podrían sugerir como indicadores de calidad de atención de enfermería: la disponibilidad y accesibilidad a servicios profesionales de enfermería, la aceptación de su acompañamiento por parte de la comunidad, los proyectos desarrollados y los logros alcanzados en mejorar la calidad de vida.

\section{Reflexiones finales}

La realidad del trabajo de los profesionales de enfermería es preocupante desde cualquier ángulo que se mire: unas condiciones de trabajo desgastantes: un proceso de trabajo anárquico, que no se compadece con las necesidades de salud de la población, ni con el costo social que representa la formación universitaria. Por otra parte, la calidad de la atención en tales condiciones - como ya lo han mostrado algunas investigacionesno puede ser más desalentadora.

Ante esta situación se requiere, mayor conciencia del problema, repensar la pertinencia de los actuales programas de formación de enfermeras, de personal auxiliar y técnicos en salud en función de la dinámica de los servicios de salud: investigación, comunicación y trabajo armónico entre el mundo de la academia y el laboral: e intervención por parte de 
las organizaciones dèenfermería, con propuestas que permitan quebrar esta historia de opresión e injusticia social. Pero, sobre todo, es necesario que cada profesional se asuma como tal. De lo contrario, estaremos perpetuando la cultura de autoritarismo y sumisión en el campo de la salud y despilfarrando tiempo y energías en programas curriculares de alto costo y bajo impacto y sometiendo a la población a riesgos permanentes en términos de la calidad de atención que recibe. 